7 Quanjer PH, Stanojevic S, Cole TJ, et al. Multi-ethnic reference values for spirometry for the 3-95-yr age range: the global lung function 2012 equations. Eur Respir J 2012; 40: 1324-1343.

8 Bourdin A, Paganin F, Prefaut C, et al. Nitrogen washout slope in poorly controlled asthma. Allergy 2006; 61: 85-89.

9 Robinson PD, Latzin P, Verbanck S, et al. Consensus statement for inert gas washout measurement using multiple- and single- breath tests. Eur Respir J 2013; 41: 507-522.

10 Burgel PR, Bourdin A, Chanez P, et al. Update on the roles of distal airways in COPD. Eur Respir Rev 2011; $20: 7-22$.

11 Hasegawa M, Nasuhara Y, Onodera Y, et al. Airflow limitation and airway dimensions in chronic obstructive pulmonary disease. Am J Respir Crit Care Med 2006; 173: 1309-1315.

12 Takahashi M, Okada H, Oguni N, et al. How accurate is CT morphometry of airway? Phantom and clinical validation study. Eur J Radiol 2011; 80: e524-e530.

\title{
Monitoring toxicity in individuals receiving treatment for latent tuberculosis infection: a systematic review versus expert opinion
}

\author{
To the Editor:
}

Latent tuberculosis infection (LTBI) is defined as a state of persistent immune response to stimulation by Mycobacterium tuberculosis antigens without evidence of clinically manifested active TB [1]. The World Health Organization (WHO) estimates that one-third of the global population is infected by Mycobacterium tuberculosis [2]. They cannot transmit tuberculosis (TB), but they can develop the disease and become infectious with a life-time risk of approximately 5-15\% [3]. A significant proportion of new TB patients, especially in low burden countries, are presumed to occur from the pool of individuals with LTBI.

Treatment of LTBI by isoniazid and/or rifamycin can reduce the risk of future disease by 60-90\% [4]. Systematic testing and treatment of LTBI is recommended in several at-risk populations with a favourable trade-off between benefits and harms of treatment. As the majority of individuals undergoing LTBI treatment would never develop $\mathrm{TB}$, regardless of therapy, it is mandatory to minimise risks during treatment. Specifically, it is imperative to design a mechanism to regularly monitor and manage adverse events.

Drug-specific adverse reactions can occur with isoniazid (asymptomatic elevation of serum liver enzyme concentrations, peripheral neuropathy and hepatotoxicity); rifampicin and rifapentine (cutaneous reactions, hypersensitivity reactions, gastrointestinal intolerance and hepatotoxicity). A meta-analysis involving 38257 subjects treated with isoniazid, estimated the risk of clinical hepatitis at six per 1000 treated individuals (range among the six studies: 0.0-2.9\%) [5]. Later, the risk of isoniazid-related symptomatic hepatitis was estimated as $1-3$, that of mortality $0-0.3$ and that of hospitalisation $0.1-0.2$ per 1000 treated persons [6]. More recently, among 24221 participants in a large trial that was conducted in South Africa, 132 possible adverse events (0.54\%) were reported among 130 individuals. Clinical hepatotoxicity was associated with the consumption of alcohol [7]. Evidence shows that the rate of adverse events, in particular death, decreased with time, probably due to better clinical care [8].

We performed a systematic review on the best clinical approach to monitor toxicity in individuals treated for LTBI to inforzm the WHO process for the development of the LTBI management guidelines [9]. The outcome measurements were: mortality and morbidity related to toxicity and tolerability of the preventive therapy. All the outcomes would have been evaluated under different proposed monitoring conditions (i.e. "wait and see", clinical and/or biochemical monitoring).

All pertinent studies, limited to English, French and Spanish languages, regardless of geographical setting, were considered. The search had no time limitations and included the following keywords combined in specific strings: TB, LTBI, toxicity, adverse events, safety, tolerability, anti-TB drugs, and preventive therapy. The search was conducted in the electronic databases PUBMED and EMBASE, and, then, expanded to the reference lists of relevant studies and review articles. All the retrieved records were electronically collected and selected on the basis of pre-defined inclusion criteria i.e. study design (cross-sectional, case-control, and retrospective/prospective cohort studies or clinical trials), type of participants (individuals with suspected LTBI, tuberculin skin test and/or interferon- $\gamma$ positive release 
TABLE 1 Main recommendations on interventions for clinical and laboratory monitoring of individuals receiving treatment for latent tuberculosis infection

Intervention Recommendation Country [ref]

Clinical monitoring At baseline and at monthly intervals during treatment.

\section{Laboratory assessment at baseline ${ }^{\#}$}

\section{Laboratory assessment during treatment ${ }^{\#}$} aged $>35$ years. risk factors" risk factors ${ }^{+}$. and those with risk factors $\S^{\S}$. for those with risk factors ${ }^{f}$.
Only recommended for individuals who are candidates for treatment

Only recommended for individuals who are candidates for treatment with

Only recommended for individuals who are candidates for treatment with

Only recommended for individuals who are candidates for treatment aged $>35$ years

Suggested for individuals who are candidates for treatment aged $>14$ years.

Recommended for all individuals who are candidates for treatment $>35$ years and

In case of symptoms: if aged 35-50 years, systematic testing should occur once at completion at 1 month. Monthly testing for all those aged $>50$ years who have risk factors $\#$

In case of symptoms and for those with baseline abnormal values, otherwise monthly for those with risk factors".

In case of symptoms, or monthly for all those aged $>35$ years, those with abnormal baselines values for a liver function test, or those with risk factors ${ }^{+}$.

Every 2-4 weeks for those with $\mathrm{HBeAG}$ positivity.

Only recommended at 2 and 4 weeks after the start of therapy and monthly thereafter for those aged $>65$ years and those with risk factors ${ }^{\text {Iा? }}$.

Monthly for those aged $>35$ years or with risk factors ${ }^{\S}$.

Systematic testing of all those receiving treatment twice during the first month, monthly thereafter in adults and every second month in children.

Some experts recommend testing every $2-4$ weeks for the first 2-3 months of treatment.
Canada [11], USA [12, 13], France [14], Portugal [15], Sweden [16], Ireland [17]

Canada [11]

USA (CDC) [12]

ATS (ATS) [13]

Portugal [15]

Ireland [17]

Canada [11]

USA (CDC) [12]

USA (ATS) [13]

USA (ATS) [13]

France [14]

Portugal [15]

Sweden [16]

Ireland [17]

HBeAG: a hepatitis B antigen protein. ${ }^{\#}$ : includes measurements of transaminases and bilirubin in all guidelines. In addition, references [16, 17] include complete blood counts in case of rifampicin treatment, and reference [13] include: screening for viral hepatitis in intravenous drug users, born in endemic areas, HIV-infected, sexual or household contact with chronically infected, occupational exposure, chronic haemodialysis, recipients of clotting factors before 1987, have undiagnosed liver disease, are recipients of blood or solid organ transplants before 1992, and are infants born to infected mothers. " : risk factors in reference [12] include: liver disorders, history of liver disease le.g. hepatitis B or C, alcoholic hepatitis, or cirrhosis), regular use of alcohol, risks for chronic liver disease, HIV infection, pregnancy, immediate post partum period, and exposure to drugs for chronic medical conditions. ${ }^{+}$: risk factors in reference [13] include: possible liver disorders, history of chronic liver disease, regular use of alcohol, HIV infection treated with highly active antiretroviral therapy, pregnancy, immediate post partum period, and exposure to drugs for chronic diseases. ${ }^{\S}$ : risk factors in reference [15] include: HIV infection, regular use of alcohol, pregnancy, immediate post partum period, liver disorders, and exposure to drugs for chronic diseases. ${ }^{f}$ : risk factors in reference [17] include: HIV infection, regular use of alcohol, pregnancy, immediate post partum period, history of hepatitis, liver disease or heavy alcohol ingestion, i.v. drug use, and treatment with other potential hepatotoxic agents. ${ }^{\# \text { : }}$ risk factors in reference [11] include: pregnancy or first 3 months post partum, history of previous drug-induced hepatitis, current cirrhosis or chronic active hepatitis of any cause, hepatitis $\mathrm{C}$, hepatitis B with abnormal transaminases, daily alcohol consumption or concomitant treatment with other hepatotoxic drugs (e.g. methotrexate). "17: risk factors in reference [14] include: regular use of alcohol, other liver disorders, poor nutritional status, and history of chronic liver disease (including viral hepatitis infection).

assay), types of interventions (“wait and see” approach, clinical monitoring, biochemical monitoring, clinical and/or biochemical monitoring), and exclusion criteria, i.e. type of manuscripts (letters, editorials, commentaries, case- or series-reports, and publications not written in English, French and Spanish). Findings were reported in accordance with the PRISMA (preferred reporting items for systematic reviews and meta-analyses) statement in an ad hoc electronic form [10].

After removing duplicates from the 102 titles identified, 43 records were screened. Only two full text articles were finally assessed for eligibility, both of which were excluded because they did not focus on monitoring approach comparison. In conclusion, our search failed to identify any study providing direct evidence on best practices for clinical monitoring of LTBI treatment.

Therefore, we reviewed 12 purposefully selected national LTBI guidelines from low-incidence countries, which were issued after 2003. Five guidelines (UK, Germany, Switzerland, Spain and Japan) were excluded 
from further analysis due to the lack of information on monitoring adverse events. A summary of the recommendations from the remaining seven guidelines [11-17] is reported in the table 1 .

Interventions to monitor adverse events were grouped into three major categories: clinical monitoring, laboratory assessment at baseline and laboratory monitoring during treatment. However, it was noted that the recommendations were consistently based on expert opinion rather than direct evidence from clinical trials.

All the seven guidelines [11-17] consistently recommend baseline evaluation with information on potential undesired drug events, monthly interaction between healthcare providers and the individual on treatment either through physical visit or telephone contact. Additionally, individuals on treatment should inform healthccare providers should there be signs or symptoms such as jaundice, abdominal pain, nausea or fever, which should result in physical examination and investigation of liver transaminases and bilirubin. Baseline evaluation should include information on potential undesired drug events (table 1).

Routine baseline laboratory testing prior to starting treatment is only recommended for selected subgroups of candidates to treatment. Some guidelines (e.g. Canada, Portugal, and Ireland) recommend baseline testing in all subjects aged $\geq 35$ years $[11,15,17]$. Guidelines from the USA recommend baseline testing only in the presence of risk factors $[12,13]$, whereas guidelines from Portugal and Ireland use both risk factors and age $[15,17]$. The risk factors described in the guidelines were not consistent (table 1).

Laboratory evaluation during treatment is usually recommended in cases where signs or symptoms described above were found and for individuals with abnormal baseline liver function test results. In addition, several guidelines recommend systematic testing at monthly intervals for selected individuals, the criteria being again age and the presence of risk factors (table 1).

The findings of this comprehensive review demonstrate that a relevant clinical and public health issue, such as clinical monitoring of individuals treated for LTBI, has not been adequately explored and standardised and largely depends on expert opinion, clinical practice and availability of resources. Decisions concerning who to prioritise for laboratory monitoring, what to monitor, and the ideal timing of monitoring are cases in point.

Toxicity monitoring may constitute a substantial proportion of the cost associated with preventive therapy, and economic, human, and financial sustainability may represent a key issue in limiting a wide implementation of programmatic LTBI management. As the newly released WHO guidelines on LTBI management are likely to result in scaling up of the treatment of LTBI, particularly in resource-rich and low-TB incidence settings, it is imperative that programmes design strategies for the implementation of science research to identify the best and cost-effective approach for clinical monitoring of adverse events.

0 @ERSpublications

LTBI treatment: a systematic review on the best clinical approach to monitor the adverse events of preventive therapy http://ow.ly/HyjAH

Giovanni Sotgiu ${ }^{1,5}$, Alberto Matteelli ${ }^{2,5}$, Haileyesus Getahun ${ }^{2}$, Enrico Girardi ${ }^{3}$, Monica Sañé Schepisi ${ }^{3}$, Rosella Centis $^{4}$ and Giovanni Battista Migliori ${ }^{4}$

${ }^{1}$ Clinical Epidemiology and Medical Statistics Unit, Dept of Biomedical Sciences, University of Sassari-Research, Medical Education and Professional Development Unit, AOU Sassari, Sassari, Italy. ${ }^{2}$ Global TB Programme, World Health Organization, Geneva, Switzerland. ${ }^{3}$ Clinical Epidemiology Unit, Dept of Epidemiology and Preclinical Research, National Institute for Infectious Diseases, IRCCS L. Spallanzani, Rome, Italy. ${ }^{4}$ World Health Organization Collaborating Centre for Tuberculosis and Lung Diseases, Fondazione S. Maugeri, Care and Research Institute, Tradate, Italy. ${ }^{5}$ Both authors contributed equally.

Correspondence: Giovanni Battista Migliori, WHO Collaborating Centre for TB and Lung Diseases, Fondazione S. Maugeri, Care and Research Institute, via Roncaccio 16, 21049, Tradate, Italy. E-mail: giovannibattista.migliori@fsm.it

Received: Nov 242014 | Accepted after revision: Dec 162014 | First published online: Feb 192015

Conflict of interest: Disclosures can be found alongside the online version of this article at erj.ersjournals.com

\section{References}

1 Mack U, Migliori GB, Sester M, et al. LTBI: latent tuberculosis infection or lasting immune responses to M. tuberculosis? A TBNET consensus statement. Eur Respir J 2009; 33: 956-973.

2 Dye C, Scheele S, Dolin P, et al. Consensus statement. Global burden of tuberculosis: estimated incidence, prevalence, and mortality by country. JAMA 1999; 282: 677-686.

3 Comstock GW, Livesay VT, et al. The prognosis of a positive tuberculin reaction in childhood and adolescence. Am J Epidemiol 1974; 99: 131-138.

4 Leung CC, Rieder HL, Lange C, et al. Treatment of latent infection with Mycobacterium tuberculosis: update 2010. Eur Respir J 2011; 37: 690-711.

5 Steele MA, Burk RF, DesPrez RM. Toxic hepatitis with isoniazid and rifampin. A meta-analysis. Chest 1991; 99: 465-471. 
6 Nolan CM, Goldberg SV, Buskin SE. Hepatotoxicity associated with isoniazid preventive therapy: a 7-year survey from a public health tuberculosis clinic. JAMA 1999; 281: 1014-1018.

7 Grant AD, Mngadi KT, van Halsema CL, et al. Adverse events with isoniazid preventive therapy: experience from a large trial. AIDS 2010; 24: Suppl. 5, S29-S36.

8 Snider DEJr, Caras GJ. Isoniazid-associated hepatitis deaths: a review of available information. Am Rev Respir Dis 1992; 145: 494-497.

9 World Health Organization. Guidelines on the Management of Latent Tuberculosis Infection. Geneva, World Health Organization, 2014. Available from: www.who.int/tb/publications/ltbi_document_page/en/

10 Moher D, Liberati A, Tetzlaff J, et al. Preferred reporting items for systematic reviews and meta-analyses: the PRISMA statement. PLoS Med 2009; 6: e1000097.

11 Public Health Agency of Canada. Canadian Tuberculosis Standards 7th Edition 2013. 2014. Ottawa, ON, Centre for Communicable Diseases and Infection Control, Public Health Agency of Canada, 2014. Available from: www. respiratoryguidelines.ca/tb-standards-2013

12 Centers for Disease Control and Prevention. Latent Tuberculosis Infection: A Guide for Primary Health Care Providers. Atlanta, GA, Centers for Disease Control and Prevention, 2013. Available from: www.cdc.gov/tb/ publications/ltbi/pdf/TargetedLTBI.pdf

13 Saukkonen JJ, Cohn DL, Jasmer RM, et al. An official ATS statement: hepatotoxicity of antituberculosis therapy. Am J Respir Crit Care Med 2006; 174: 935-952.

14 Haut Conseil de la Santé Publique. Enquête Autour d'un Cas de Tuberculose: Recommandations Pratiques. [SurveyAround a Case of Tuberculosis Practical Recommendations]. Paris, Haut Conseil de la santé publique, 2013. Available from: www.hcsp.fr/Explore.cgi/Telecharger?NomFichier=hcspr20131025_enquetecastuberculoserecoprat.pdf

15 Sociedade Portuguesa de Pneumologia. Tratamento da Tuberculose Latente. Revisão Das Normas [Treatment of Latent Tuberculosis. Review of Rules]. Lisbon, Sociedade Portuguesa de Pneumologia, 2006. Available from: www. dgs.pt/documentos-e-publicacoes/tratamento-da-tuberculose-latente.aspx

16 Socialsyrelson. Rekommendationer för preventiva insatser mot tuberkulos - hälsokontroll, smittspårning och vaccination [Recommendations for preventive measures against tuberculosis - health surveillance, contact tracing and vaccination]. www.socialstyrelsen.se/smittskydd/sjukdomar/smittsammasjukdomarochsmittamnen/tuberkulos Date last accessed: November 11, 2014. Date Last updated: March 01, 2012.

17 Health Protection Surveillance Centre. Guidelines on the Prevention and Control of Tuberculosis in Ireland 2010 Dublin, Health Protection Surveillance Centre, 2010. Available from: www.hpsc.ie/AboutHPSC/ScientificCommittees/ Publications/File,4349,en.pdf

\section{A novel whole-blood miRNA signature for a rapid diagnosis of pulmonary tuberculosis}

To the Editor:

One essential aspect for controlling the spread of tuberculosis (TB) is to diagnose it in an early stage. However, the commonly used test systems are still insufficient. Moreover, current assays perform poorly in discriminating between active TB and latent tuberculosis infection (LTBI). Due to limited knowledge of promising TB biomarkers, global "omics" approaches bear attractive options to follow [1]. MiRNAs are important post-transcriptional regulators shown to be involved in the modulation of immune responses against intracellular pathogens [2]. These findings open the possibility of using miRNAs as TB biomarkers for diagnosis. In the present study, we have investigated their role as blood biomarkers for detecting active TB.

Blood samples were collected from five different participating institutions located in Barcelona (Spain). We enrolled a total of 50 individuals, classified into three groups: 1) 17 LTBI individuals. They had been in close recent contact with a high exposure to a smear positive or negative pulmonary TB ( $\mathrm{pTB}$ ) patient. The inclusion criteria were a positive tuberculin skin test (TST), at least one positive result for one of the interferon (IFN)- $\gamma$ assays and no more than 2 weeks of chemoprophylaxis. 2) 17 active pTB patients with a positive culture for Mycobacterium tuberculosis and no more than 2 weeks of anti-TB therapy. 3) 16 